\title{
DEVELOPING A DATA FUSION STRATEGY BETWEEN OMNIDIRECTIONAL IMAGE AND INDOORGML DATA
}

\author{
A. R. C. Claridades ${ }^{1,2}$, D. Ahn ${ }^{1}$, J. Lee ${ }^{1 *}$ \\ ${ }^{1}$ Dept. of Geoinformatics, University of Seoul, Dongdaemun-gu, Seoul, South Korea - \{uosgrad2019012, ads903, jlee $@ @$ uos.ac.kr \\ ${ }^{2}$ Dept. of Geodetic Engineering, University of the Philippines Diliman, Quezon City, Philippines
}

\section{Commission IV}

KEY WORDS: Geospatial Data Fusion, Topological Data Model, 3D GIS, Indoor GIS, IndoorGML

\begin{abstract}
:
As the interest in indoor spaces increases, there is a growing need for indoor spatial applications. As these spaces grow in complexity and size, research is being carried out towards effective and efficient representation. Omnidirectional images give a snapshot of interiors and give visually rich content, but only contain pixel data. For it to be used in providing indoor services, its limitations must be overcome. First, the images must be connected to each other to represent indoor space continuously based on spatial relationships that may be provided by topological data. Second, the objects and spaces that we see in these images must also be recognized. This paper presents a study on how to link omnidirectional images and an IndoorGML data without the need for data conversion, provision of reference data, or use of different data models in order to provide Indoor Location-Based Service (LBS). We introduce the use of the Spatial Extended Point (SEP) to characterize the relationship between the omnidirectional image and the topological data. Position information of the object is used to define a region of 3D space, to determine the inclusion relationship of an IndoorGML node. We conduct an experimental implementation of the integrated data in the form of a 3D Virtual Tour. The connection of the Omnidirectional images is demonstrated by a visualization of navigation through a hallway towards a room's interior delivered to the user through a clicking action on the image.
\end{abstract}

\section{INTRODUCTION}

Nowadays, spatial applications move towards the indoors and as these spaces continue to be more complex the interest in applications (Giudice et al., 2010). Together with the pervasiveness of mobile technology and social media (Zheng et al., 2017), more efficient and effective representations are warranted to ensure timely, accurate, and relevant information is delivered to users.

As various researchers approach this problem differently, various forms of data have been used to represent the various aspects of the indoor environment, primarily as the representation method depends on the purpose of the intended service. These variations in data generation, management, and application becomes the source of problems in implementation due to issues of compatibility and connectivity (Ahn et al., 2018). Data fusion approaches aim to solve this problem by integrating multiple datasets to produce the same information that come from the sources. Various approaches have been explored for this purpose, such as data conversion, exploring relationships through attributes, or referring to an external reference data. In addition, these methods aim for consistency in representation in geometric and topological aspects to increase understanding of the relationships of the features themselves that are represented.

Omnidirectional images, presenting rich visual content yet having a manageable data size, may be used for indoor space modelling. However, visual content present in the images are not sufficient to provide indoor spatial applications, beyond the level of visualization. An omnidirectional image provides a 360-degree field-of-view, yet only static snapshot of a scene at the instant it was taken. To be able to represent the continuity of indoor spaces, images must be connected to each other in a certain way.

Similarly, since pixel data only gives a visualization of the spaces and there is no topological information, so the relationships between spaces are not recognized. For instance, we cannot immediately infer that a room is adjacent to another room from an image, or if a room is accessible from a particular hallway. Similarly, objects found within these spaces can be visually identified by a user but are not immediately recognized as individual features. In order for omnidirectional images to be used in providing indoor spatial applications, such as locationbased services (LBS), it must be capable of handling analysis on indoor space, just as much as geometric data. To do this, we must be able to link the images based on spatial relationships that can be provided by topological data (Kim et al., 2016). This will also be a key to identify the objects and spaces that are represented in the images.

IndoorGML is the international standard for indoor topological data, created in light of the need for representing indoor spaces in the context of navigation. Although there are other standards like CityGML and IFC that are used for 3D datasets, this standard focuses on topological relationships of spaces, represented as edges linking the nodes that represent the spaces themselves (OGC, 2015). Data fusion has been used on linking IndoorGML data with another dataset representing indoor space, but this is based on geometric information (Lee et al., 2014), or by using datasets based on other data models (Kim et al., 2013), both of which are not applicable for image data. Omnidirectional images has been linked with topological information from IndoorGML data to provide an indoor patrol service, but this approach used an external reference data that

\footnotetext{
* Corresponding author
} 
contains attribute information for the indoor spaces and objects (Jung and Lee, 2017).

In this study, we propose a data fusion method for image data with network-based topological data without relying on data conversion, use of separate data models, and an external reference data. We aim to link omnidirectional images and IndoorGML data based on spatial relationships, to recognize indoor images and provide a platform for indoor space applications. In the following section, we describe existing data fusion approaches for IndoorGML data, then in the following section the methodology is described. We conduct an experimental implementation, discussed in the fourth section and the final section summarizes the limitations of this study and areas for further research.

\section{RELATED STUDIES}

In these times, human beings rely more on mobile technology for fast delivery and retrieval of information, as exhibited by services such as social media and navigation interfaces. An instance of this are location-based services, that rely on geospatial datasets to relay information to the user depending on location (Park et al., 2017). However, across fields or application domains, representation of geographical objects that exists in the real world as geospatial datasets because they vary in purpose. This means that in many cases, there are multiple datasets that may be generated to represent the same geographic features (Park et al., 2018), and this poses problems such as compatibility, duplication and integration.

Data fusion techniques aim to bridge this problem, by providing solutions to unify datasets coming from various sources, collected using different sensors or methods, or are based on different data standards, but still provide the same, if not increased, level of information that the individual sources provide. Various approaches have been explored for this purpose, such as data conversion where one data format is exchanged to its counterpart (Teo and $\mathrm{Yu}, 2017$ ), mapping attributes to develop correspondence between features (Ok et al., 2009), developing a relation model among spatial objects (Lee et al., 2014), or using a reference data to establish relationships (Jung and Lee, 2017).

Topological information is necessary for Location BasedServices as they provide information on relationships that are crucial for data integration, analysis, and presentation. More specifically, topological analysis (such as adjacency, intersection, connectivity, containment) is supported, as well as shortest-path analyses (Gunduz et al., 2016). Ellul and Haklay (2006) classified topological data according to form as Matrixbased, Boundary-representation, and Network-based types (Ellul and Haklay, 2006)- but Lee and Lee (2010) showed that network-based models perform the best in analysis (Lee and Lee, 2010).

IndoorGML is a network-based topological data published by the Open Geospatial Consortium (OGC), based on the Noderelation structure proposed by Lee (2004). It utilizes the concept of Poincare Duality to abstract spatial entities into topological objects, so that $3 \mathrm{D}$ space such as a room, or a hallway is represented as a node, and the topological relationships between said spaces are by edges (or lines) that connect these nodes (Lee, 2004). This standard describes indoor spaces as any space within one or multiple buildings, which may or may not be enclosed by a roof. Furthermore, it describes the basic topological relationships of adjacency, connectivity, and accessibility between indoor spaces through node-relation graphs (NRG), and does not necessarily contain geometric information (OGC, 2015).

Omnidirectional images have been used across applications to represent spaces. Popular web maps such as Google Street View (Google, 2018) have used omnidirectional images to represent outdoor transportation networks for visualization, as well as interiors of major public areas (Daum, 2018; Google, 2018). Its implementation in generating IndoorGML-based topological data has been through the identification of spaces in the image through methods such as image filtering and classification (Kim et al., 2016; Kim and Lee, 2015), and through a moving window approach (Claridades et al., 2018). In both approaches, the IndoorGML notion of indoor space are applied first on how the images are collected along the interior, say at Shooting Points (Claridades et al., 2018), and how their respective topological relationships among each other are defined, the latter elaborating more on generating specific NRGs.

BIM and CAD data are widely-used to present spatial information about structures and its interiors, but they are often too complicated, hard to obtain, and not updated (Claridades et al., 2018). IndoorGML focuses on the aspect of indoor navigation, but since it has been published by the OGC, starting points for further is identified as this standard only includes minimum requirements for indoor spatial modelling. A use-case agenda on indoor routing services and extensions for common application domains is encouraged to expand its potential and application (Kang and $\mathrm{Li}$, 2017). Data fusion studies involving IndoorGML data may include data conversion, such as approaches to convert CityGML data to IndoorGML (Khan et al., 2014), or IFC to IndoorGML (Teo and $\mathrm{Yu}, 2017$ ), yet these approaches pose dangers of information loss along the way. To overcome this problem, an approach for data fusion without the need for conversion is preferred. This extends the capability of the data model across applications, and enables better data management, interoperability and sharing. Approaches for data fusion may either be through geometry, visualization, or topology. The first one is mainly used for BIM and CityGML datasets, and this requires identification of corresponding objects between source and target data, making it inapplicable for images. Visualization based approaches however, has limitations on providing seamless indoor and outdoor services (Ahn et al., 2018; Park et al., 2018).

For IndoorGML data, the data fusion approach by topology is suitable, this being a standard for topological information. An Indoor Spatial Data Model based on combining CityGML as the feature model and IndoorGML as the topology model is proposed (Kim et al., 2013), while this work has been extended conversely in the Topological Relation Model (TRM), by extending IndoorGML through defining topological relations of two datasets with corresponding geometric space models so that geometric data coming from two different datasets are used directly through matching (Lee et al., 2014), however this is developed at an application service level, and not at the data level. The Topological Relation-based Data Fusion Model (TRDFM) extends the ArchNode proposed by the IndoorGML standard by specifying the manner on how topology data may be generated from the surface-based, volume-based, and network-based data and establish connections through the topology directly (Park et al., 2018). However, topological relationships between objects and spaces cannot be inferred directly in the images as these contain only pixel data. 
To overcome this, Jung and Lee (2017) proposed an Omnidirectional image-based data model by extending the IndoorGML data model by defining an association between the CellSpace class and the Omnidirectional image. This data model was implemented by representing each node in the IndoorGML NRG as an image through a topology authoring tool. Spaces are recognized in the image since routing based on the topology data can be performed along a path along the images. In this application, however, delivery of object information relies by calculating the image coordinate and comparing this coordinate to a polygon in a reference data. The identified polygon will then be used to select which corresponding Node representing an IndoorGML State, the contained information of which will be displayed to the user (Jung and Lee, 2017).
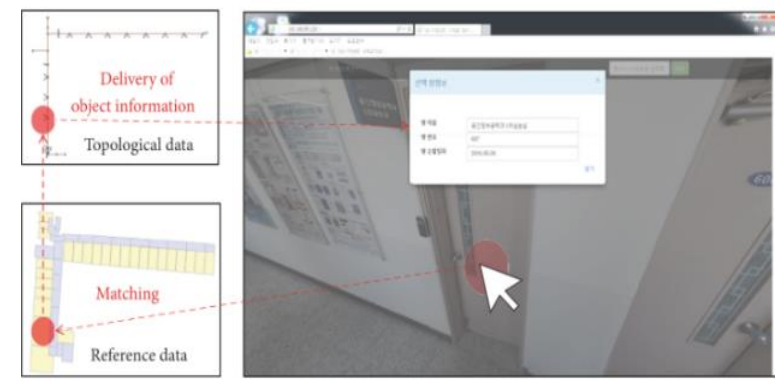

Figure 1. Delivery of object information in Omnidirectional image from Topological data through a Reference data (Jung and Lee, 2015)

In the same study, the Indoor Patrol Service Platform is built upon the base of discretely-connected omnidirectional images, where each image shows a 360 -degree view about a point, so navigating to another location will remove the image from view and load another omnidirectional image (Jung and Lee, 2017). While expressing connectivity between spaces, the corresponding adjacency is not expressed despite being provided by the topological data. This dilutes the information on the continuity of indoor space, as it is discretized by the images per Shooting Point. In addition, in order to move to the next image, a fiducial mark placed on the floor where the next shooting point is located in the image must be clicked by the user, restricting movement along the space since the user can only click on that one point in order to navigate.

In the goal of providing an efficient means to calculate topological relationships between spatial objects, the 9intersection mode was defined for points, lines, and polygons. As an integrated model defines these relationships as an approach for optimizing spatial queries as opposed to more computationally-demanding methods such as an R-tree (Clementini et al., 1994).

This concept was discussed further in the context of spatially extended topology for moving objects by using the 9intersection model to define a topological relationship between a point and an area. The defined matrix from Lee (2009) is shown as Equation (1), defining the $3 \times 1$-matrix $M_{p}$ representing region-point relationships, based on the point object $\left(\mathrm{P}^{\circ}\right)$, and an interior $\left(R^{\circ}\right)$, boundary $(\partial R)$, and exterior $\left(R^{-}\right)$of an region.

$$
\mathrm{M}_{\mathrm{p}}=\left[\begin{array}{lll}
\mathrm{P}^{\circ} \cap \mathrm{R}^{\circ} & \mathrm{P}^{\circ} \cap \partial \mathrm{R} & \mathrm{P}^{\circ} \cap \mathrm{R}^{-}
\end{array}\right]
$$

This is applied in a model representing topological relationships of objects and their respective range of influence (Lee, 2009). In this paper, we demonstrate the use of this concept in providing a data fusion method for an Omnidirectional Image and IndoorGML data.

\section{METHODOLOGY}

In this section, we first describe the data requirements for image and topological data fusion, and then we discuss the proposed method for establishing a connection between the image and topological data.

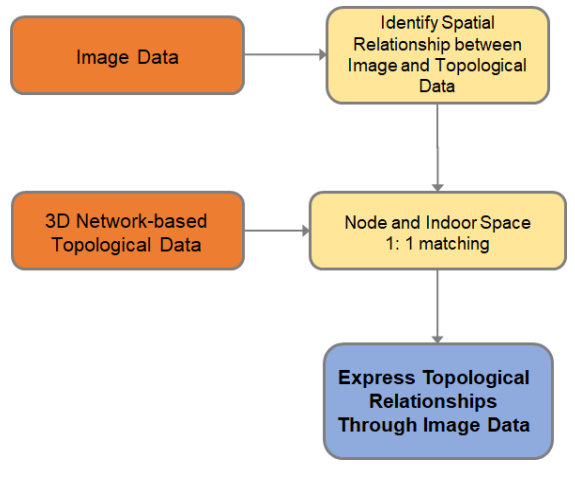

Figure 2. Image and Topological Data Fusion Framework

Figure 2 shows the framework for the data fusion approach involving image data and a 3D Network-based topological data. Image data is a suitable representation method for indoor space because of its simplicity and high visual content. To supplement information and make it suitable for providing LBS, it will be integrated with a topological data based on a 3D network structure. This process will be based on the spatial relationships, so a 1:1 match can be identified for the indoor spaces- those detected in the image and those that are represented as nodes in the topological data. With this, the topological relationships provided by the topological data can now be expressed directly through the image data.

\subsection{Data Requirements for Image and Topology Data Fusion}

The main purpose of the data fusion is to provide a platform for location-based services in the indoor environment. With this, the image data to be used for this purpose should be able to represent indoor space continuously. We use an omnidirectional image to visualize the indoor space, as well as the objects contained in these spaces. Apart from being able to portray surroundings in three dimensions, it entails less construction costs than the use of geometric data which makes data update less expensive, both in terms of time, cost and computation.

Since omnidirectional images are snapshots taken at a certain fixed location, i.e., Shooting Points, the set if images representing a continuous space should be connected to each other through spatial relationships. Location-based services using these images as a platform also require these spaces to be recognizable, as well as the objects that are contained in these spaces.

Representing indoor space by omnidirectional images alone may not be enough in some applications, such as navigation which requires visualization of movement, since 
omnidirectional images can only provide the 360-degree view around the Shooting Point. We use IndoorGML data to represent the spatial relationships that provide the basis for the connections of the images. Indoor LBS requires information on adjacency and connectivity of spaces, which can be readily provided by IndoorGML. Omnidirectional images, together with this information, will represent indoor space more accurately since this will.

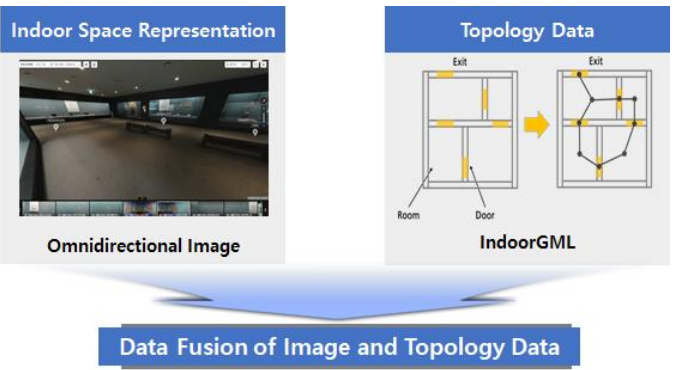

Figure 3. Data Requirements for Data Fusion

3.1.1 Building the Omnidirectional Image Data: Omnidirectional images must be taken at Shooting Points located along the interior space, identified by the IndoorGML node. As shown by Claridades et al. (2018), this is the most practical location of shooting points because apart from maximizing the space hence minimizing the number of images, a correspondence between the image and IndoorGML data can be easily defined. Since multiple omnidirectional images may be taken in a large space, such as a corridor, the directional reference for the omnidirectional image may vary depending on how the image is stitched during the acquisition process, or how the camera captured the scene. In both cases, the heading of the image must be adjusted to the same direction, as shown in Figure 4.

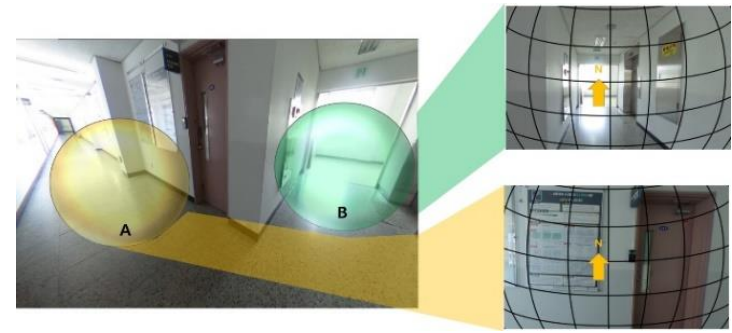

Figure 4. Consistency of Image Headings

3.1.2 Constructing the IndoorGML Data: IndoorGML abstracts 3D space as 0-dimensional nodes, and the respective topological relationships as 1-dimensional edges between the nodes, based on the Poincare Duality. With this, the simple network structure can express the complexity of 3D space easily.

To provide a more realistic navigation service, the door of each room is treated as a separate indoor space, because a person must pass through the space provided by the door in order to go inside or outside the room. Figure 5 demonstrates the connectivity and adjacency relationships of rooms R1, R2, and R3 among each other. In addition, their respective relationships with the door D1 are also shown, as defined by IndoorGML. R1 is adjacent to R2 and R3, respectively, but is not connected directly- a person navigating must pass through the door first before being located outside or inside this room. It is for this reason that there are edges that connect R1 and R3, and R1 and $\mathrm{R} 2$ in the adjacency graph, but there are none in the connectivity graph.
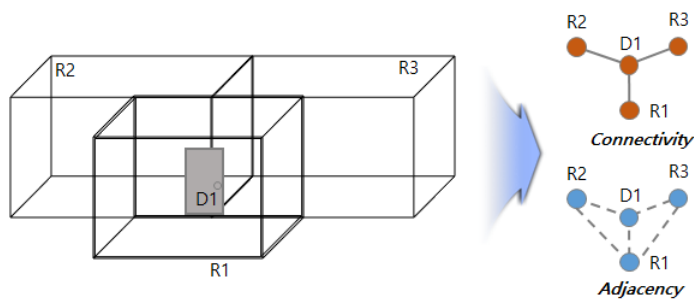

Figure 5. IndoorGML Topological Relationships

Since IndoorGML data is composed of nodes and the images are composed of pixels, there is a difficulty in establishing a 1:1 matching for the purpose of identifying indoor space in the image. The image-based topology authoring tool produces an XML file structure, each image is indexed to a scene, where connections to the other images are expressed as calls for a Krpano script to load another scene. However, for the purpose data fusion where further topological relationships and recognition of objects, this is not enough. To overcome this, topology data based on IndoorGML is constructed as an XML database and is integrated to the image XML file. Figure 6 shows the structure for each connected node to an image.

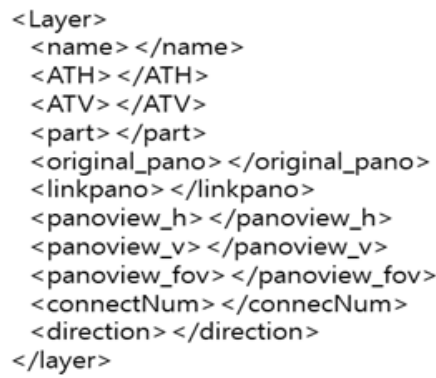

Figure 6. Topology data in XML structure

In addition to the name, parameters that are necessary to provide a navigation service is included. These are position information (i.e., the horizontal angle and vertical angle at which the connected node is located in the image), viewing parameters (field of view, default look-to directions), number of nodes, function of the node (i.e., space or door) and direction of navigation.

\subsection{Establishing a connection based on relationships between Omnidirectional Image and Indoor Topological Data}

Since IndoorGML data is composed of nodes and the images are composed of pixels, there is a difficulty in establishing a one is to one (1:1) matching for the purpose of identifying indoor space in the image. To do this, we need to define the relationship of the nodes with the spaces. The identification of indoor space in the omnidirectional images, as represented by pixels, begins with the user input, the mouse-click. The threedimensional position of the pixel will be calculated using 
horizontal and vertical angles. The pseudocode of this algorithm is shown in Figure 7.

\begin{tabular}{|c|c|}
\hline \multicolumn{2}{|c|}{ getCoordinate(mouse_ath, mouse_atv, r, offset) } \\
\hline in & $\begin{array}{l}\text { mouse_ath, mouse_atv : angle of object in scene } \\
\mathbf{r}: \text { omnidirectional Image radius } \\
\text { offset : Camera height }\end{array}$ \\
\hline out & $x_{12} y_{12} z_{1}$ : Coordinate Selected Point \\
\hline & $\begin{array}{l}\text { Click_mouse : check click } \\
\text { Angle_H : Horizontal angle } \\
\text { Angle_V : Vertical angle } \\
\mathbf{r}: \text { Omnidirectional Image radius } \\
\text { offset : Camera height } \\
\text { Hd : Heading } \\
\text { Begin } \\
\text { IF click_mouse is False } \\
\text { IF directional of Camera is directly North, Hd } \leftarrow 0 \\
\text { Angle_H }=(\text { Hd }+ \text { mouse_ath) } * \mathrm{PI} / 180 \\
\text { Angle_V = mouse_atv *PI/180 } \\
\boldsymbol{x}_{1}=\mathrm{r} * \cos (\text { Angle_H) } \\
y_{1}=\mathrm{r} * \sin (\text { Angle_H) } \\
z_{1}=\text { offset }+\mathrm{r} * \sin (\text { Angle_V) } \\
\text { click_mouse set TRUE }\end{array}$ \\
\hline
\end{tabular}

Figure 7. Pseudocode for calculating coordinates

To calculate the 3D coordinates, the angular positions of the mouse click, the omnidirectional image radius, and the height are camera during capture is used. If the direction of the camera (user's view) is directly north, the heading is set to zero, and increases clockwise. This heading value is added to the horizontal angle and is used to obtain the $\mathrm{x}$ and $\mathrm{y}$ coordinates, while the vertical angle and the offset is used for the $\mathrm{z}$ coordinate.

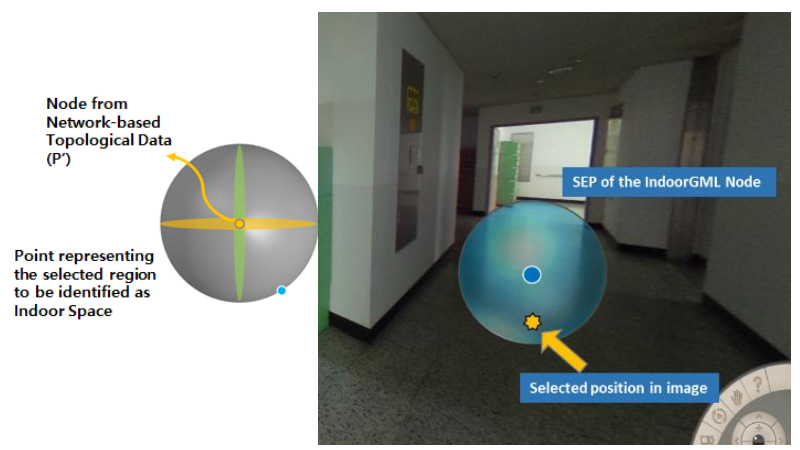

Figure 8. The Spatial Extended Point concept applied to identifying topological relationships

To identify an indoor space referred to by the user once a position in the image is selected, the corresponding IndoorGML node representing the indoor space must be identified through the use the concept of the Spatial Extended Point (SEP) which will define a region in space around which the space is identified, and the point object- in this case is the IndoorGML node. This is illustrated by Figure 8. To achieve this, we use an SEP matrix to represent topological relationships between the image and the space. From Equation (1), we extend the region in $3 \mathrm{D}$, define the matrix in Equation (2), where a value of 1 is assigned upon satisfying the condition and 0 otherwise.

$$
\left[\begin{array}{lll}
P^{0} \cap R_{x y}, & P^{0} \cap \partial R_{x y} & P^{0} \cap R_{x y}{ }^{-} \\
P^{0} \cap R_{y z} & P^{0} \cap \partial R_{y z} & P^{0} \cap R_{y z}{ }^{-} \\
P^{0} \cap R_{x z} & P^{0} \cap \partial R_{x z} & P^{0} \cap R_{x z}-
\end{array}\right]
$$

where $\quad P^{0}=$ Selected point in image

$R_{x y}{ }^{\circ}=$ Inside the boundary along the XY plane

$R_{y z}=$ Inside the boundary along the $\mathrm{YZ}$ plane

$R_{X z}=$ Inside the boundary along the XZ plane

$\partial R_{x y}=$ Lies on the boundary along the XY plane

$\partial R_{y z}=$ Lies on the boundary along the YZ plane

$\partial R_{x z}=$ Lies on the boundary along the XZ plane

$R_{X y}{ }^{-}=$Outside the boundary along the XY plane

$R_{y z}{ }^{-}=$Outside the boundary along the YZ plane

$R_{X z}{ }^{-}=$Outside the boundary along the XZ plane

The SEP region $(R)$ is defined from the position of the IndoorGML node, as well as the positions of the user click $(P)$. To calculate values for the matrix, the $3 \mathrm{D}$ region representing the SEP will be projected in to the corresponding three $2 \mathrm{D}$ cartesian planes, and for each plane, the positions of the IndoorGML nodes will be used to identify if it is inside, outside or lying on the boundary of the region. The pseudocode in Figure 9 is used for this process.

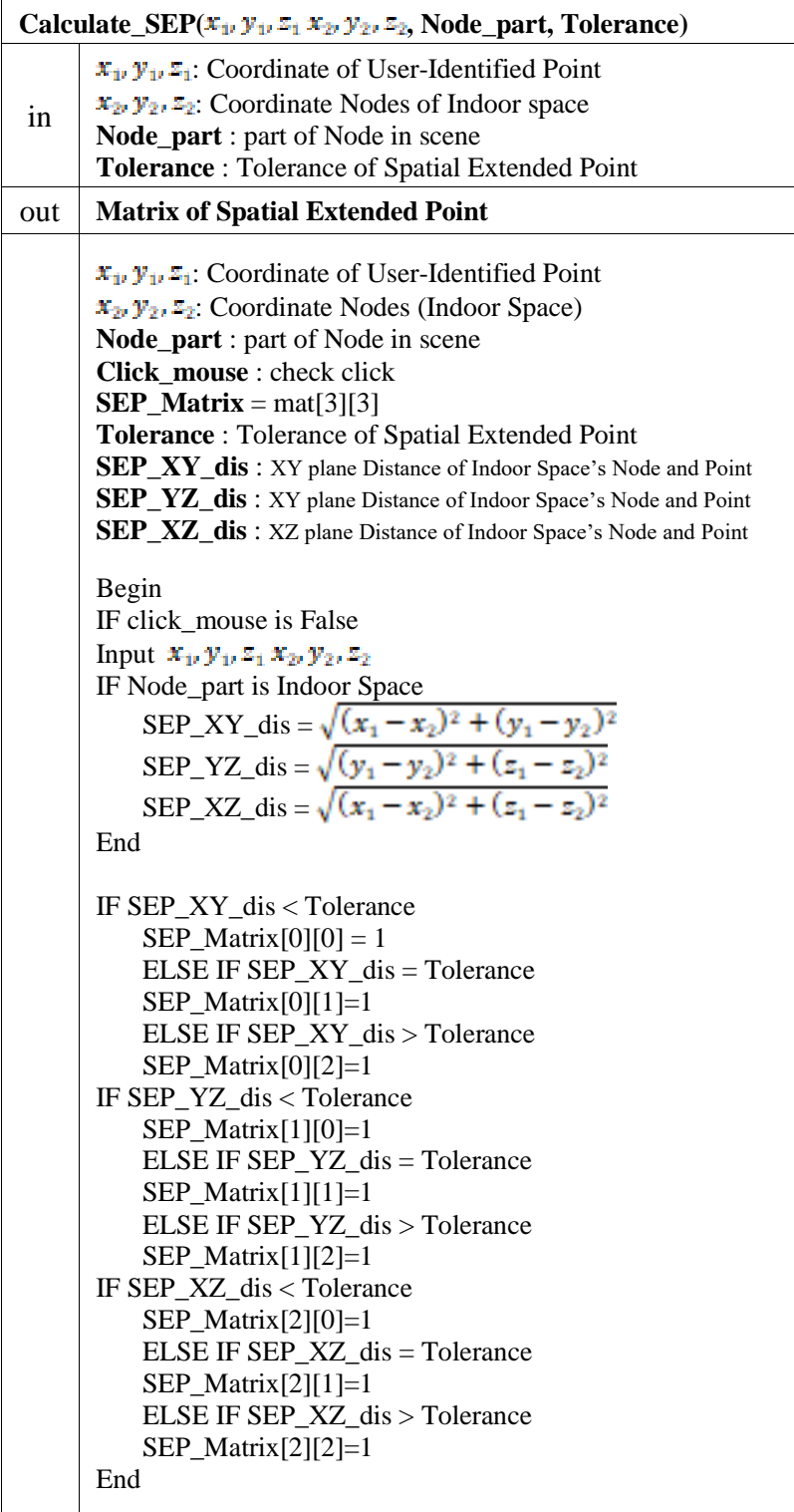

Figure 9. Pseudocode for the SEP Matrix calculation 
Since relationships that can now be inferred from the connection between the image and topological data using the SEP, indoor spaces can now be identified in the image. In this regard, by identifying the indoor space in an image by clicking on a location (i.e., a pixel), the associated omnidirectional image for the identified space in that location can be visualized, and this process can be used for a navigation application. For instance, double-clicking a space in the omnidirectional image, we find out which IndoorGML node would correspond to the SEP of that space. Figure 10 illustrates the pseudocode for this process.

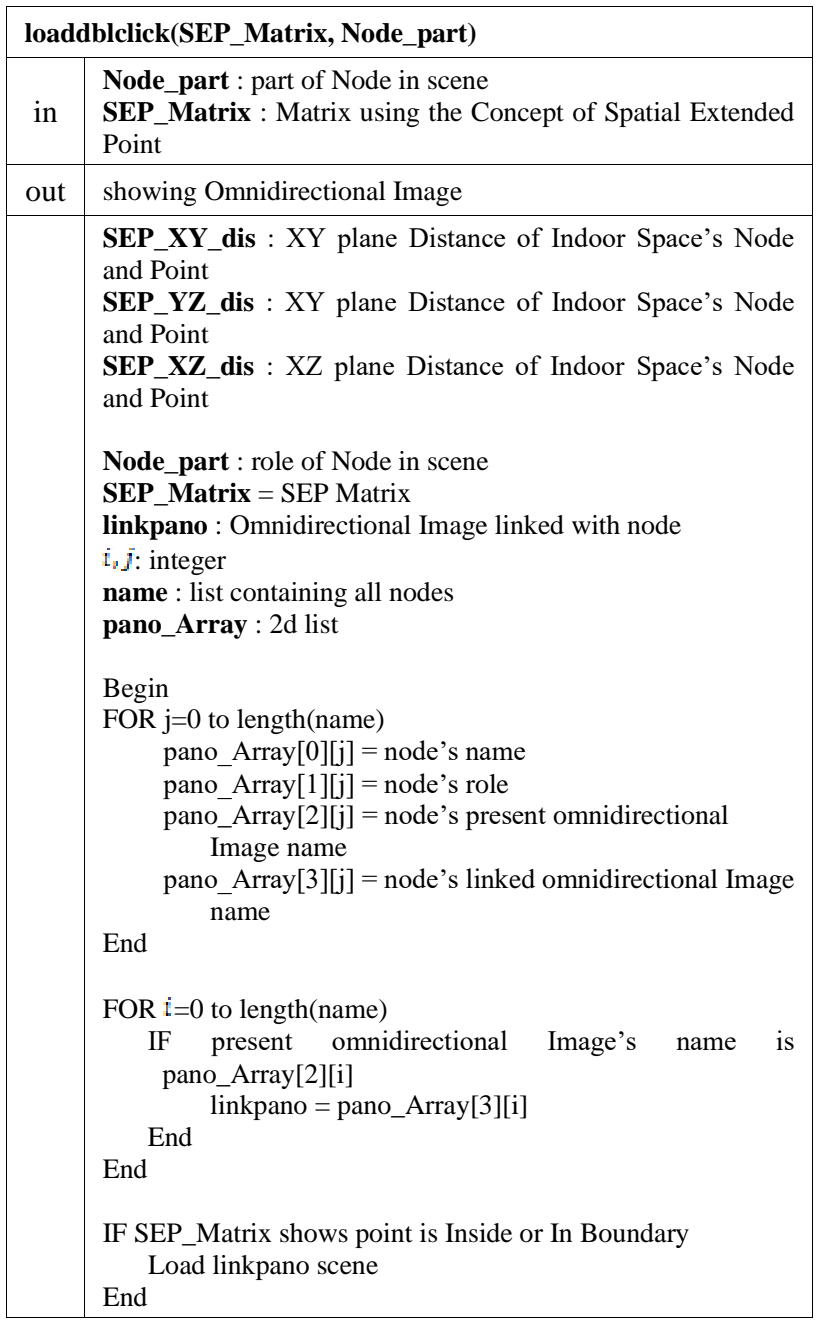

Figure 10. Pseudocode for navigation using SEP concept

Based on Figure 10, since relationships that can now be inferred from the connection between the image and topological data using the SEP, indoor spaces can now be identified in the image. In this regard, by a user action such as double clicking, the user can move to that identified space, presented visually as a movement from one omnidirectional image to another.

\section{EXPERIMENTAL IMPLEMENTATION}

In this section, we demonstrate the proposed methodology by building a visualization platform of the image data based on Omnidirectional Images and IndoorGML data fusion. The study area for the experimental implementation was the $6^{\text {th }}$ floor of the 21st Century Building of the University of Seoul, South Korea (Figure 11).

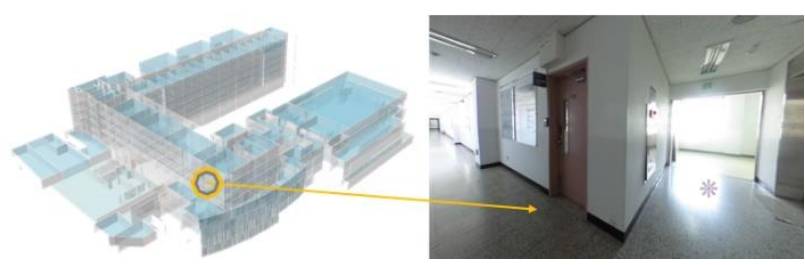

Figure 11. Study Area

In the study area, photographs were taken at each shooting point using a DSLR camera mounted on a rotator with a fisheye lens, and a Ricoh Theta S. Images were stitched in PTGui to construct the omnidirectional images, and were connected through the image-based topology authoring tool PanoTour Pro 2.5 that produced HTML and XML Files for the service. In addition, Bitnami was used to build a local server to handle the integrated image and topology data. Web-based languages including HTML, JavaScript, and XML were used to provide the services in the implementation of the data fusion methodology. These are summarized in Table 1.

\begin{tabular}{|l|c|}
\hline Image Shooting Equipment & $\begin{array}{c}35 \text { mm DSLR camera (36.4 } \\
\text { million pixels) } \\
180^{\circ} \text { fisheye lens } \\
\text { Rotary rotator } \\
\text { Ricoh Theta S (12 million } \\
\text { pixels) }\end{array}$ \\
\hline $\begin{array}{l}\text { Omnidirectional Image } \\
\text { Stitching Program }\end{array}$ & PTGui 10.0.12 \\
\hline $\begin{array}{l}\text { Image-based Topology } \\
\text { Authoring Tool }\end{array}$ & Kolor PanoTour Pro 2.5 \\
\hline Web Server & Bitnami (Apache) \\
\hline Server Script & XML, HTML, JavaScript \\
\hline JavaScript Plugin & Krpano \\
\hline
\end{tabular}

Table 1. Experimental Environment

In order to generate the topological data used in this study, the experimental environment as shown in Figure 12 below must be satisfied. The IndoorGML data is represented as the XML structure shown in Figure 6, together with the references to the file directories containing the images.

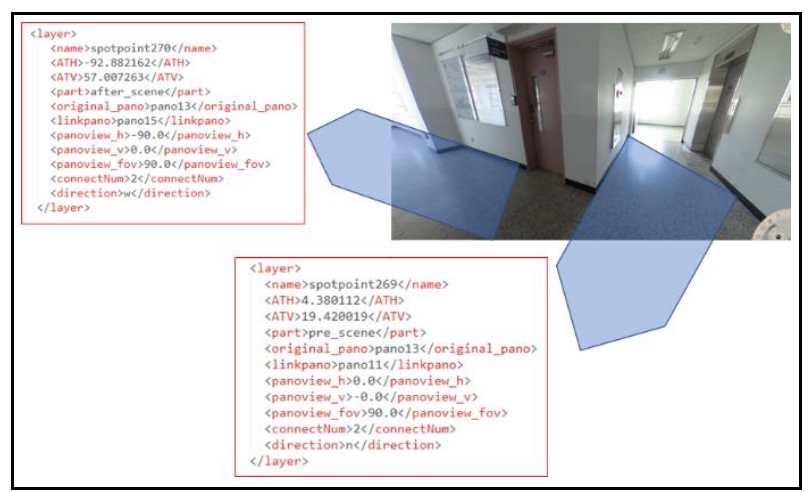

Figure 12. Configuring attributes of the topological data nodes for the areas present in the image in the XML file

The pseudocodes presented in Figure 7, 9, and 10 were coded in JavaScript as the internal functions of the experiment, while the HTML code provided the integration of the XML and 
JavaScript files, and addition of basic user-interface elements. This platform capable of being operated using an ordinary webbrowser, as shown in Figure 13.

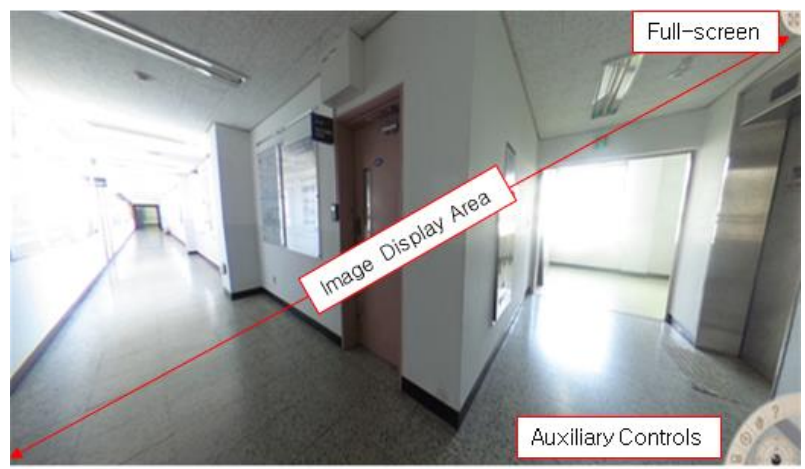

Figure 13. Basic interface of the demo program

The user can pan, zoom, and scroll around a singe point, the position being the shooting point of the omnidirectional image, both using the mouse and the auxilliary controls. A full-screen display option is also available.
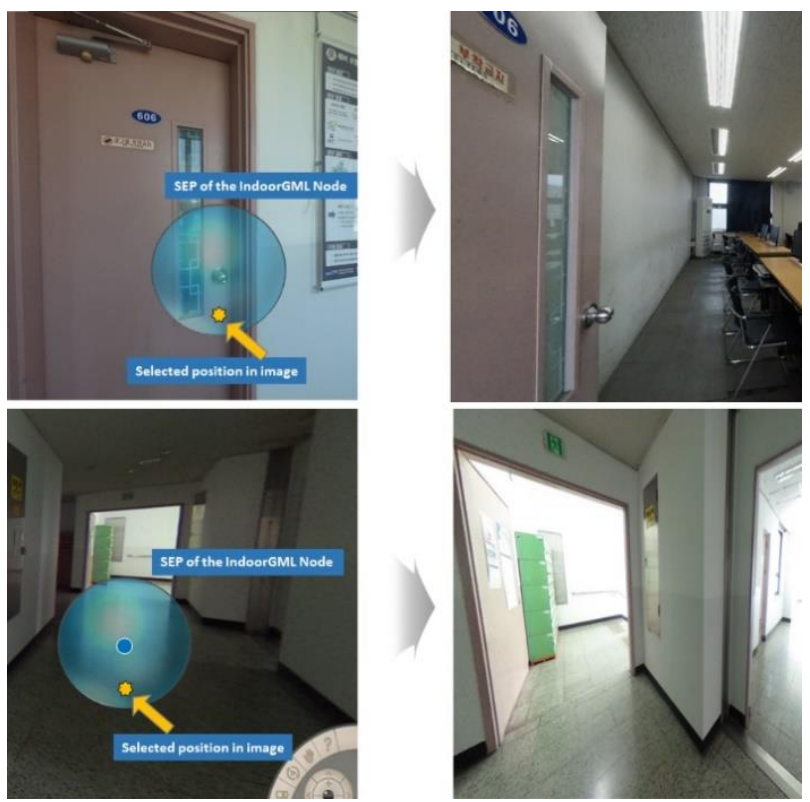

Figure 14. Visualization of omnidirectional image upon recognition of space in current image

Figure 14 shows the case of identifying a space in the omnidirectional image, and then displaying the corresponding omnidirectional image of the space identified, based on the IndoorGML node included in the SEP of the mouse click location. The top image shows a case of navigation from a hallway going towards a room, where a mouse click is placed on the doorknob in the image, where the user is guided towards the image with an open door in the next node's position, ready to move inside the room. The bottom image shows the navigation in a hallway, where a forward motion is visualized upon clicking a pixel ahead of the user, and the following omnidirectional image based on the IndoorGML node in that direction is displayed. In addition to this, the transition from image to image is smoothly, impressing the idea that indoor space is continuous thorugh the topological relationships from the IndoorGML data, despite the omnidirectional image being collected discretely along shoting points in the hallways and rooms.

\section{CONCLUSIONS}

Various techniques have been made available by previous studies in representing indoor space, and focus is given to topological datasets that allow for efficient and accurate spatial analysis. Omnidirectional images present a realistic way to visualize indoor space, plus having low construction cost and being easier to update shows promise in its utility in indoor space applications. However, since it lacks geometry and topology, additional data is necessary to provide information to users in indoor services such as LBS. This paper proposes a data fusion method between omnidirectional images and topological data using the concept of Spatial Extended Point.

The topological data used to express indoor space and their respective relationships is IndoorGML, a standard established by OGC. The integration of IndoorGML data to the omnidirectional image is vital as this enables spaces within the images to be recognized among the pixel data. Using the concept of the SEP, a user-identified position based on a pixel can be used to define a region of influence, where a possible IndoorGML node is located- which then corresponds to an indoor space. We perform an experimental implementation in the interior of a university building, where omnidirectional images are collected, and an application performing navigation from image to image. This also further demonstrates the continuity of indoor space in contrast to the images alone, which are collected discretely along the interior.

This study has some limitations that future studies may have to address. First, objects located in the indoor space must also be recognized apart from the indoor spaces themselves. However, they are not modelled explicitly by IndoorGML which primarily concerns indoor spaces. While previous studies have addressed this by modelling these objects as POI and defining topological relationships with the indoor spaces, there is a need to integrate this information with how data fusion with omnidirectional images and topological data is performed.

In addition, indoor topological data constructed in this study is based on a corridor-type indoor space. Because of this, a different case topological data may be involved in other types of indoor spaces, such as those that are wider or those that directly connect to the outdoors. Considering these matters would generalize the case of the fusion of omnidirectional image and indoor topological data.

\section{ACKNOWLEDGEMENTS}

This research was supported by a grant(19NSIP-B135746-03) from National Spatial Information Research Program (NSIP) funded by Ministry of Land, Infrastructure and Transport of Korean government.

\section{REFERENCES}

Ahn, D., Park, J., Lee, J., 2018. Defining geospatial data fusion methods based on topological relationships. Int. Arch. Photogramm. Remote Sens. Spat. Inf. Sci. - ISPRS Arch. 42, 317-319. https://doi.org/10.5194/isprs-archives-XLII-4-W9- 
Claridades, A.R., Lee, J., Blanco, A., 2018. Using Omnidirectional Images for Semi-Automatically Generating IndoorGML Data. J. Korean Soc. Surv. Geod. Photogramm. Cartogr. 36, 319-333. https://doi.org/https://doi.org/10.7848/ksgpc.2018.36.5.319

Clementini, E., Sharma, J., Egenhofer, M., 1994. Modeling Topological Spatial Relations: Strategies for Query-Processing. Comput. Graph. 18, 815-822.

Daum, 2018. Daum Store View [WWW Document]. URL http://map.daum.net/?nil_profile=title\&nil_src=local (accessed 4.13.18).

Ellul, C., Haklay, M., 2006. Requirements for Topology in 3D GIS. Trans. GIS 10, 157-175. https://doi.org/10.1111/j.14679671.2006.00251.x

Giudice, N.A., Walton, L.A., Worboys, M., 2010. The informatics of indoor and outdoor space: A research agenda. Proc. 2nd ACM SIGSPATIAL Int. Work. Indoor Spat. Aware. (ISA 2010) 47-53. https://doi.org/http://dx.doi.org/10.1145/1865885.1865897

Google, L., 2018. Google Street View [WWW Document]. URL https://www.google.com/streetview/understand/ (accessed 4.13.18).

Gunduz, M., Isikdag, U., Basaraner, M., 2016. A Review of Recent Research in Indoor Modelling \& Mapping. Int. Arch. Photogramm. Remote Sens. Spat. Inf. Sci. - ISPRS Arch. 41, 289-294. https://doi.org/10.5194/isprsarchives-XLI-B4-2892016

Jung, H., Lee, J., 2017. Development of an OmnidirectionalImage-Based Data Model through Extending the IndoorGML Concept to an Indoor Patrol Service. J. Sensors 2017. https://doi.org/10.1155/2017/5379106

Jung, H., Lee, J., 2015. Indoor Subspacing to Implement IndoorGML for Indoor Navigation. Int. Arch. Photogramm. Remote Sens. Spat. Inf. Sci. - ISPRS Arch. 40, 25-27. https://doi.org/10.5194/isprsarchives-XL-2-W4-25-2015

Kang, H.K., Li, K.J., 2017. A Standard Indoor Spatial Data Model-OGC IndoorGML and Implementation Approaches. ISPRS Int. J. Geo-Information 6, 116. https://doi.org/10.3390/ijgi6040116

Khan, A.A., Donaubauer, A., Kolbe, T.H., 2014. A Multi-Step Transformation Process for Automatically Generating Indoor Routing Graphs from Existing Semantic 3D Building Models. 9th 3DGeoInfo Conf. 2014 - Proc.

Kim, M., Kang, H.Y., Lee, J., 2016. A Study on the Implementation of Indoor Topology Using Image Data. J. Korean Soc. Surv. Geod. Photogramm. Cartogr. https://doi.org/10.7848/ksgpc.2016.34.3.329

Kim, M., Lee, J., 2015. Developing a Method to Generate IndoorGML Data from the Omni-directional Image. Int. Arch. Photogramm. Remote Sens. Spat. Inf. Sci. - ISPRS Arch. 40, 17-19. https://doi.org/10.5194/isprsarchives-XL-2-W4-17-2015

Kim, Y.J., Kang, H.Y., Lee, J., 2013. Development of Indoor
Spatial Data Model using CityGML ADE. Int. Arch. Photogramm. Remote Sens. Spat. Inf. Sci. - ISPRS Arch. XL2/W2, 41-45. https://doi.org/10.5194/isprsarchives-XL-2-W241-2013

Lee, B., 2009. Location Based Concierge Service with Spatially Extended Topology for Moving Objects. Spat. Inf. Res. 17, 445-454.

Lee, J., 2004. A Spatial Access-Oriented Implementation of a 3D GIS Topological Data Model for Urban Entities. Geoinformatica 15, 1-7.

Lee, J., Kang, H.Y., Kim, Y.J., 2014. Developing Data Fusion Method for Indoor Space Modeling based on IndoorGML Core Module. J. Korea Spat. Inf. Soc. 22, 31-44. https://doi.org/10.12672/ksis.2014.22.2.031

Lee, S., Lee, J., 2010. Efficient Topological Data Models for Spatial Queries in 3D GIS. Geospatial Data Geovisualization Environ. Secur. Soc. Spec. Jt. Symp. ISPRS Comm. IV AutoCarto 2010 conjunction with ASPRS/CaGIS 2010 Spec. Conf. XXXVIII.

OGC, 2015. OGC® IndoorGML. Open Geospatial Consort. 117. https://doi.org/http://www.opengeospatial.org/

Ok, J., Hee, W., Kiyun, Y., 2009. Matching Method of Digital Map and POI for Geospatial Web Platform. Korean Soc. Geospatial Inf. Syst. 17, 23-29.

Park, J., Ahn, D., Lee, J., 2018. Development of Data Fusion Method Based on Topological Relationships Using IndoorGML Core Module. J. Sensors 2018, 1-14. https://doi.org/10.1155/2018/4094235

Park, J., Kang, H.Y., Lee, J., 2017. A Spatial-temporal POI Data Model for Implementing Location-based Services. J. Korean Soc. Surv. Geod. Photogramm. Cartogr. 34, 609-618. https://doi.org/10.7848/ksgpc.2016.34.6.609

Teo, T.A., Yu, S.C., 2017. The Extraction of Indoor Building Information from BIM to OGC IndoorGML. Int. Arch. Photogramm. Remote Sens. Spat. Inf. Sci. - ISPRS Arch. 42, 167-170. https://doi.org/10.5194/isprs-archives-XLII-4-W2$167-2017$

Zheng, Z., Chen, Y., Chen, S., Sun, L., Chen, D., 2017. Location-aware POI recommendation for indoor space by exploiting WiFi logs. Mob. Inf. Syst. 2017. https://doi.org/10.1155/2017/9601404 